\title{
Recurrent Neural Processing and Somatosensory Awareness
}

\author{
Ryszard Auksztulewicz, ${ }^{1}$ Bernhard Spitzer, ${ }^{2,3}$ and Felix Blankenburg ${ }^{2,3}$ \\ ${ }^{1}$ Berlin School of Mind and Brain, Humboldt-Universität zu Berlin, 10099 Berlin, Germany, ${ }^{2}$ Department of Neurology, Charité-Universitätsmedizin Berlin, \\ 10117 Berlin, Germany, and ${ }^{3}$ Bernstein Center for Computational Neuroscience, 10115 Berlin, Germany
}

The neural mechanisms of stimulus detection, despite extensive research, remain elusive. The recurrent processing hypothesis, a prominent theoretical account of perceptual awareness, states that, although stimuli might in principle evoke feedforward activity propagating through the visual cortex, stimuli that become consciously detected are further processed in feedforward-feedback loops established between cortical areas. To test this theory in the tactile modality, we applied dynamic causal modeling to electroencephalography (EEG) data acquired from humans in a somatosensory detection task. In the analysis of stimulation-induced event-related potentials (ERPs), we focused on model-based evidence for feedforward, feedback, and recurrent processing between primary and secondary somatosensory cortices. Bayesian model comparison revealed that, although early EEG components were well explained by both the feedforward and the recurrent models, the recurrent model outperformed the other models when later EEG segments were analyzed. Within the recurrent model, stimulus detection was characterized by a relatively early strength increase of the feedforward connection from primary to secondary somatosensory cortex $(>80 \mathrm{~ms})$. At longer latencies $(>140 \mathrm{~ms})$, also the feedback connection showed a detection-related strength increase. The modeling results on relative evidence between recurrent and feedforward model comparison support the hypothesis that the ERP responses from sensory areas arising after aware stimulus detection can be explained by increased recurrent processing within the somatosensory network in the later stages of stimulus processing.

\section{Introduction}

Recurrent activity loops between hierarchical stages of sensory processing play an important role in perception. There is substantial evidence for the involvement of recurrent processing in visual processes, such as figure-ground segregation, curve tracing, and attentive grouping (Lamme and Roelfsema, 2000). More recently, recurrent dynamics between cortical areas have been used to model evoked neuronal responses to auditory stimuli in a mismatch paradigm (Garrido et al., 2007).

Parallel to studies on basic perceptual processes, recurrent processing has gained popularity as a candidate mechanism underlying perceptual consciousness, with evidence ranging from studies using transcranial magnetic stimulation-induced phosphenes (Pascual-Leone and Walsh, 2001), to visual masking (Ro et al., 2003; Boehler et al., 2008; Fahrenfort et al., 2008), motion detection tasks (Silvanto et al., 2005; but see Koivisto et al., 2010), and effective connectivity analyses in patients with consciousness disorders (Boly et al., 2011). As a result, several influential views on consciousness treat recurrent activity as a condition necessary (Dehaene et al., 2006; Tononi and Koch, 2008) or even sufficient (Lamme, 2000, 2006) for perceptual awareness.

Received Aug. 3, 2011; revised 0ct. 26, 2011; accepted Nov. 21, 2011.

Author contributions: R.A. and F.B. designed research; R.A. and B.S. performed research; R.A. and B.S. analyzed data; R.A., B.S., and F.B. wrote the paper.

This work was supported by a grant from the German Federal Ministry of Education and Research (F.B.). We thank Timo Schmidt and Evelin Wacker for help with data acquisition, as well as Peter Frensch, Robert Gaschler, Dirk Ostwald, and Will Penny for helpful advice.

The authors declare no competing financial interests.

Correspondence should be addressed to Ryszard Auksztulewicz, Berlin School of Mind and Brain, Luisenstraße 56, Haus 1, 10099 Berlin, Germany. E-mail: ryszard.auksztulewizz@gmail.com.

DOI:10.1523/JNEUROSCI.3974-11.2012

Copyright $\odot 2012$ the authors $\quad 0270-6474 / 12 / 320799-07 \$ 15.00 / 0$
To generalize findings from vision, it is crucial to study mechanisms underlying detection in other modalities. The somatosensory system, given the relatively large spatial separation of primary and secondary cortices, seems suitable for testing the role of local cortical interactions, and extensive previous research on poststimulus correlates of somatosensory awareness makes touch an excellent candidate for corroborating findings from vision. However, because somatosensory research typically focused on the latency of earliest observable neuronal responses (Libet et al., 1967; Ray et al., 1999; Schubert et al., 2006) and the characterization of oscillatory activity (Meador et al., 2002; Palva et al., 2005), the possible role of recurrent processing for tactile detection remains poorly understood.

Indirect evidence for neuronal feedback in tactile perception came from single-neuron recordings in macaques (de Lafuente and Romo, 2006) detecting weak mechanical stimulation, in which the analysis of response latencies of single neurons across the recorded areas, from early somatosensory areas to motor cortex, revealed a large latency overlap. Although the indication that some individual neurons from higher cortical areas responded faster than other neurons from lower areas was consistent with the recurrent processing hypothesis, the role of such neuronal feedback in tactile detection has not been tested directly.

Dynamic causal modeling (DCM) allows to study the neuronal architecture underlying observed electromagnetic signals and the effective connectivity between its sources, making it a useful tool in testing alternative models of causal interactions between brain areas that explain the measured data. In this study, we applied DCM to electroencephalography (EEG) data (David et al., 2006) acquired in a somatosensory detection task, assessing evidence for feedforward, feedback, and recurrent 
processing between primary (SI) and secondary (SII) somatosensory cortices. We tested whether recurrent processing in the model is needed to explain the differences in EEG responses between detected and missed stimuli. We hypothesized that, although early EEG responses can be explained by feedforward processing alone, explaining later ERP components will require both feedforward and feedback connections in the model.

\section{Materials and Methods}

Participants. Twenty-three healthy individuals (14 female; aged 18-32 years; mean $\pm \mathrm{SD}$, $25.3 \pm 3.7$ years) participated in the experiment after giving written informed consent. All participants had normal or corrected-tonormal vision. The experimental procedure was approved by the ethics committee of Charité Medical School of Berlin.

Stimuli and behavioral task. Single electrical constant-current pulse stimuli $(0.2 \mathrm{~ms}$ duration) were applied transcutaneously to the left median nerve (DS3; Digitimer) at detection threshold. Detection threshold was estimated before administering the task using a staircase procedure.

The detection task consisted of 600 trials divided into three blocks. After a $500 \mathrm{~ms}$ fixation period, a visual cue was displayed and remained on the screen for 1500 ms. In $80 \%$ of the trials, perithreshold stimuli (mean \pm SD intensity, $2.3 \pm 0.56 \mathrm{~mA}$ ) were applied with random delay 100-1400 ms after cue onset. In the remaining $20 \%$ trials, no stimuli were presented (catch trials). Participants indicated responses by pressing one of two buttons with their right index and middle fingers (response-button mapping randomized across participants) after the visual stimulus disappeared. The maximum response interval duration was set to $2 \mathrm{~s}$, and no feedback was given. The stimulation intensity was readjusted after each block to effective detection threshold.

After the detection task, an additional localizer run was administered. The localizer consisted of 800 suprathreshold stimuli (mean \pm SD intensity, $6.7 \pm 3.35 \mathrm{~mA}$ ) delivered with $2 \mathrm{~Hz}$ frequency and random $0-10 \mathrm{~ms}$ jitter. The intensity was adjusted to just below the muscle twitch level and, if this was reported as uncomfortable, to just below subjective threshold for unpleasantness.

EEG recording and analysis. EEG signals were recorded using a 64channel active electrode system (ActiveTwo; BioSemi). The electrodes were placed in an elastic cap according to 10-10 system, and their locations were registered using an electrode positioning system (CMS20S; Zebris Medical). EEG signals were digitized at $2048 \mathrm{~Hz}$ and downsampled offline to $512 \mathrm{~Hz}$. High-pass $(>1 \mathrm{~Hz})$ and stop-band (48-52 $\mathrm{Hz})$ filters were applied to continuous data. Signals were referenced against average reference. Analyses were performed using the SPM8 EEG/MEG software package (Wellcome Trust Centre for Neuroimaging, Institute of Neurology, University College London, London, UK) and custom MATLAB code (MathWorks).

Localizer preprocessing and dipole fitting. The localizer data were epoched into segments ranging between -50 and $200 \mathrm{~ms}$ relative to stimulus onset and baseline corrected to prestimulus period. Eye movement artifacts were rejected by careful visual inspection (on average, $11.34 \pm$ $8.04 \%$ trials rejected, mean $\pm \mathrm{SD}$; average maximum retained amplitude, $63.73 \mu \mathrm{V})$. Data from one participant showing strong $\alpha$-like artifact, which resulted in rejecting $\sim 50 \%$ trials, were excluded from the analysis. Data from three additional participants were discarded from the analysis as a result of very poor $\left(D^{\prime}<0.5\right)$ or very high $\left(D^{\prime}>2.4\right)$ behavioral performance in the detection task. The remaining data $(n=19)$ were averaged across trials using weighted averaging. To provide initial loca- tion priors for subsequent dipole fitting, 3D source reconstruction (as implemented in SPM8) was performed per participant (default settings for 12 participants; for the remaining seven participants, as a result of relatively strong peristimulus artifacts, custom settings included reconstruction without the Hanning window and were restricted to the poststimulus period). Source reconstructions were inspected for two time windows centered at the individual N20 latency ( $22 \mathrm{~ms}$ after stimulus on average) and 90-115 ms after stimulus (Hagiwara et al., 2010). Representative voxels were identified for contralateral SI and bilateral SII on the basis of the two time windows, respectively, within the anatomically correct locations of a given region (determined using cytoarchitectonic probabilistic maps from SPM Anatomy Toolbox; Eickhoff et al., 2005).

Equivalent current dipoles were fitted to localizer data using the variational Bayes scheme (Kiebel et al., 2008) and using negative free energy as the approximation to model evidence (or log-evidence, i.e., the probability of observing the data given a model; Stephan et al., 2009). The following procedure was run per participant, fitting the dipoles iteratively 10 times at each step and selecting the dipole model with maximum negative free energy (Fig. 1): (1) single SI dipole was fitted to data at the individual N20 latency (22 ms after stimulus on average), with location priors set to coordinates from individual 3D source reconstruction (prior location variance, $10 \times 10 \times 10 \mathrm{~mm}$ ); and (2) a symmetrical SII dipole pair was added to the optimized SI dipole and fitted to data within the 90-115 ms time window (Hagiwara et al., 2010), with location priors from the respective $3 \mathrm{D}$ source localization and identical prior location variance (Thees et al., 2003). SII was chosen to be modeled as a symmetrical dipole pair based on its bilateral activation in the 3D source reconstruction of the 90-115 ms time window (group inversion, $10 \mathrm{~mm}$ smoothing; right SII, MNI (50, -4, 14); left SII, MNI (-52, -28, 12); both $p_{\mathrm{FWE}}<0.01$ peak level] as well as on previous evidence for its bilateral involvement in processing sensory information from either hand (Disbrow et al., 2001).

ERP analysis. Data from the detection task were segmented into 500ms-long epochs between -100 and 400 ms relative to stimulus onset and baseline corrected to prestimulus period. Eye movement artifacts were rejected after visual signal inspection $(9.48 \pm 11.24 \%$ trials rejected on average, mean $\pm \mathrm{SD}$; maximum amplitude retained, $66.04 \mu \mathrm{V})$. Data were averaged across trials for detected and undetected stimuli sepa- 
rately. Mean signals were low-passed filtered at $48 \mathrm{~Hz}$. ERPs were tested for significant differences between "detected" and "missed" trials using SPM-implemented second-level paired $t$ tests on interpolated scalp images per time point, while correcting for multiple comparisons using the false discovery rate (FDR) method (Benjamini and Hochberg, 1995; Fahrenfort et al., 2007), which fixes the proportion of expected false positives to value $\alpha_{\mathrm{FDR}}$ (here $\alpha_{\mathrm{FDR}}=0.05$ ) by sorting the $p$ values of $m$ tests on $H_{1, \ldots, m}$ null hypotheses in ascending order, finding the largest $k$ such that $p_{k} \leq \frac{k}{m} \alpha_{\mathrm{FDR}}$, and rejecting all hypotheses $H_{1, \ldots, k}$. Having identified significant effects in scalp-time space, single channels were inspected in sensor space using a series of within-subjects $t$ tests ( $\alpha=$ $0.05, \alpha_{\mathrm{FDR}}=0.01$ ).

$D C M$. We used DCM to infer hidden parameters of neuronal models from the epoched EEG data acquired in the detection task (David et al., 2006). DCM explains the data by a network model with a few dynamically interacting sources. The sources, here implemented as equivalent current dipoles, can be (1) connected in a fixed manner, modeling context-independent effective connectivity between regions, (2) exerting context-dependent influence on one another, representing modulatory connections, and (3) receiving direct driving input.

All structural models in the subsequent analyses were specified for the same architecture including a single contralateral (i.e., right) SI dipole (cSI) and a symmetrical SII dipole pair (cSII and iSII). Peripheral input was assumed to be directly received only by cSI. The models contained a bilateral structural connection between cSI and cSII and a bilateral connection between cSII and iSII. The models differed with respect to the number of individual extrinsic connection strengths modulated by condition, i.e., allowed different subsets of connections to change the strength of coupling between regions depending on stimulus detection (see Fig. $3 A$ ). A null model was also specified, containing the same structural architecture as the other models and no extrinsic connection modulation.

In the first step, to identify the optimal modulation pattern of the cSII-iSII connection, four models with a fixed cSI-cSII connection (without modulation) and varying cSII-iSII connection (bilateral, ipsilateral-to-contralateral, contralateral-to-ipsilateral, and no modulation) were computed per participant. Trials in which stimuli were missed were treated as baseline. The stimulus input onset was set to $20 \mathrm{~ms}$, modeling the N20 component. The four models were compared using a fixed-effects Bayesian model selection procedure (BMS; Stephan et al., 2010), which chooses the best model based on the log evidence (approximating the probability of observing the data given a model) pooled across single participants. Fixed-effects BMS was performed for 11 data segments separately, all starting at $1 \mathrm{~ms}$ and with length ranging from 40 $\mathrm{ms}$ (allowing to include the $\mathrm{N} 20$ component) to $240 \mathrm{~ms}$ by steps of $20 \mathrm{~ms}$ (Garrido et al., 2007).

The cSII-iSII modulation of the model winning in most data segments was then implemented in three models constructed to test effective connectivity between cSI and cSII. The models, representing feedforward, feedback, and recurrent processing, were fitted to detection data using DCM (SPM8) and compared by a fixed-effects BMS for the same 11 data segments as in the first model optimization step. The latency of cSI activation was again set to $20 \mathrm{~ms}$, and the "undetected" condition was treated as baseline.

The winning model from the latter comparison was further analyzed for trial-specific effects on the strength of each connection. The connection strength for each connection and trial type was extracted from each DCM using Bayesian model averaging (BMA) for every data segment. The connection strengths for missed stimuli were calculated as an exponential of the BMA parameters in the endogenous connectivity matrix and for detected stimuli as an exponential of the sum of BMA parameters in the endogenous connectivity matrix and the respective mean modulatory parameters.

\section{Results}

\section{Behavioral results}

The subjects $(n=19)$ detected on average $50.26 \pm 12.9 \%$ stimuli (mean $\pm \mathrm{SD}$ ) and correctly rejected $90.41 \pm 7.88 \%$ catch trials, resulting in mean $\pm \mathrm{SD} D^{\prime}=1.41 \pm 0.52$ and mean $\pm \mathrm{SD}$ bias $C=0.70 \pm 0.26$. A repeated-measures ANOVA revealed no significant changes in detection rate across the three detection blocks $\left(F_{(2,38)}=1.77, p=0.18\right)$. Mean response times did not differ across conditions $(2 \times 2$ repeated-measures ANOVA on individual mean response times after cue offset; factors: stimulus presentation and stimulus report; all $F<1.5$, all $p>0.3$ ). Averaged over participants $(n=19)$ and conditions, the mean \pm SD response time after cue offset was $528 \pm 98 \mathrm{~ms}$.

\section{Event-related potentials}

The grand-averaged EEG response from the localizer $(n=19)$ showed expected somatosensory evoked potential components (Allison et al., 1991), including a clearly pronounced centroparietal N20 (peak amplitude relative to neighboring time points at electrode CP4, 21 ms after stimulus; mean amplitude relative to adjacent time points, $\left.-0.45 \mu \mathrm{V} ; t_{(1,18)}=-3.99, p<0.001\right)$, followed by P25 and N35 components (Fig. 1).

In the detection task, the ERPs closely matched findings from previous studies (Schubert et al., 2006), following the same dynamics for both detected and missed stimuli for the first $\sim 75 \mathrm{~ms}$ and showing a divergence of EEG responses between detected and missed stimuli only for the later components (Fig. 2D). The centrally distributed contralateral N140 component was the first to reliably differ between detected and undetected stimuli (second-level paired $t$ tests as implemented in SPM, corrected for multiple comparisons using FDR at $\alpha_{\mathrm{FDR}}=0.05$, cluster peaking at $135 \mathrm{~ms}, q_{\mathrm{FDR}}=0.02$ peak level; Fig. 2 A). Looking across single channels, the N140 component peaked at electrode C6, $138 \mathrm{~ms}$ after stimulus (peak difference: $t_{(1,18)}=-5.41, p<0.001$; within-subjects paired $t$ test per time point; significant differences for all consecutive time points, 121-225 ms; $p<0.05$, $\alpha_{\mathrm{FDR}}=0.01$; Fig. $2 B$ ), although an earlier significant ERP divergence was observed, peaking at $80 \mathrm{~ms}\left(t_{(1,18)}=-5.01, p<0.001\right)$. A broadly distributed P300-like positive deflection, present after stimulus detection, peaked at electrode $\mathrm{FCz}, 363 \mathrm{~ms}$ after stimulus $\left(t_{(1,18)}=4.81, p<0.001\right.$; Fig. $\left.2 C\right)$.

\section{DCM}

In the first step, fixed-effects BMS (group level, $n=19$; Stephan et al., 2010) was used to identify the model with the most likely modulation pattern for the cSII-iSII connection. Four models with fixed cSI-cSII connection and varying cSII-iSII connection strength (four possibilities of modulation: fixed connection, ipsilateral-to-contralateral modulation, contralateral-to-ipsilateral modulation, and bilateral modulation) were compared for 11 data segments, all starting at $1 \mathrm{~ms}$ and with length ranging from 40 to $240 \mathrm{~ms}$ in steps of $20 \mathrm{~ms}$. The model with bilateral cSII-iSII connection modulation showed the highest log evidence across all data segments longer than $40 \mathrm{~ms}$ (for the $40 \mathrm{~ms}$ segment, it was outperformed by the model with contralateralto-ipsilateral modulation allowed), and therefore this modulation pattern was chosen as optimal for subsequent analyses.

In the second step, the null, feedforward, feedback, and recurrent models were compared using BMS (group level, $n=19$, fixed effects). The null model had lower evidence than the remaining three models for all data segments. Furthermore, the feedback model had lower evidence than the feedforward and recurrent models for all time segments but one $(240 \mathrm{~ms}$, for which it outperformed the feedforward model; Fig. 3B). Turning to a direct comparison of the recurrent and feedforward models, both explained data equally well for data segments shorter than $120 \mathrm{~ms}$ (difference in log evidence < 5). Crucially, for longer data 
A
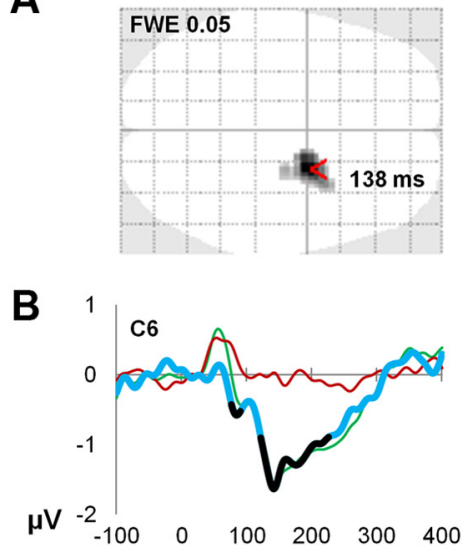

C

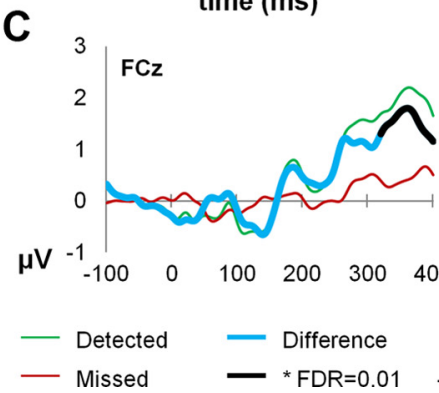

D
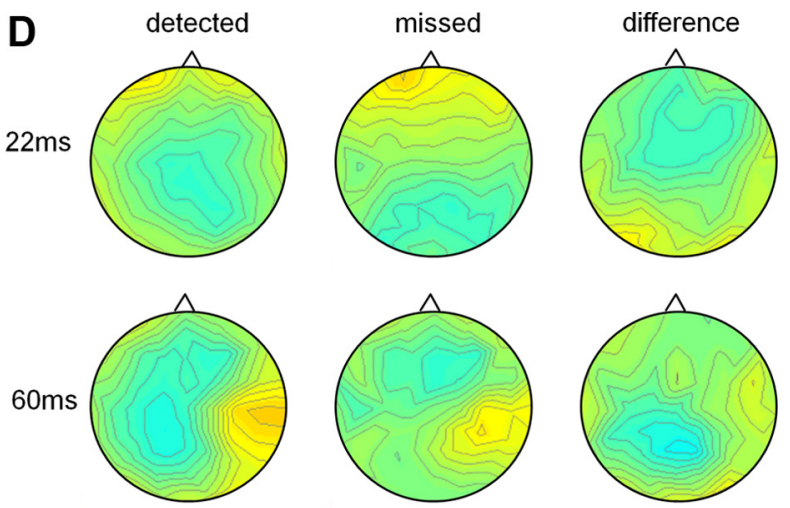

$140 \mathrm{~ms}$
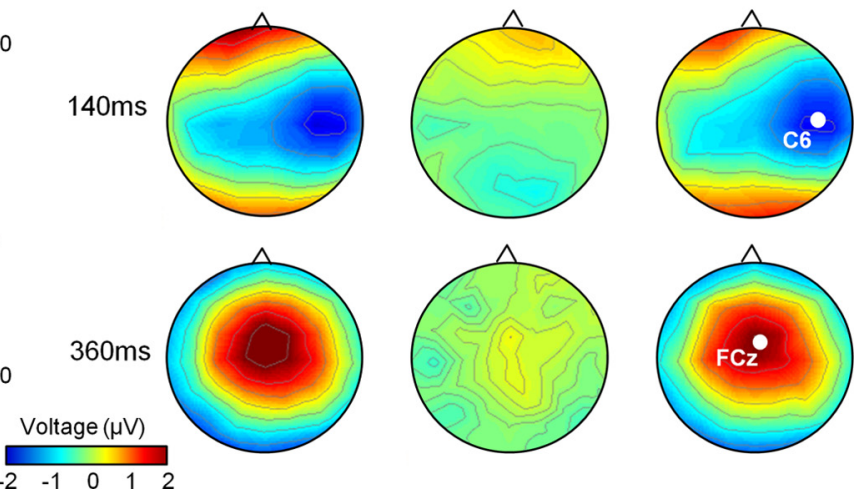

Figure 2. A, N140 component distribution. EEG responses showed late divergence for detected and undetected stimuli peaking at $135 \mathrm{~ms}$ within a central contralateral cluster (second-level paired $t$ tests, corrected for multiple comparisons using FDR at $\alpha_{\mathrm{FDR}}=0.05, q_{\mathrm{FDR}}=0.02$ peak level). B, C, ERPs characterizing tactile detection. N140 amplitude was largest at electrode (6. A broadly distributed P300-like component peaked at electrode FCz. D, Topographical maps. Grand-averaged voltage distributions are shown on topographical maps for detected and missed targets at four latencies corresponding to typical somatosensory ERP components (N20, P60, N140, and P300, respectively).

A

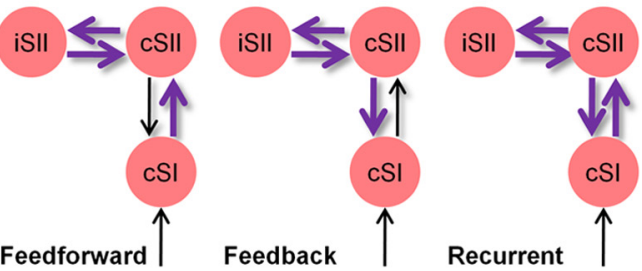

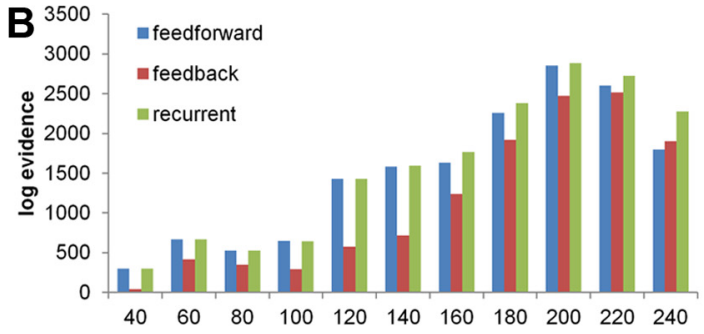

epoch length (ms)

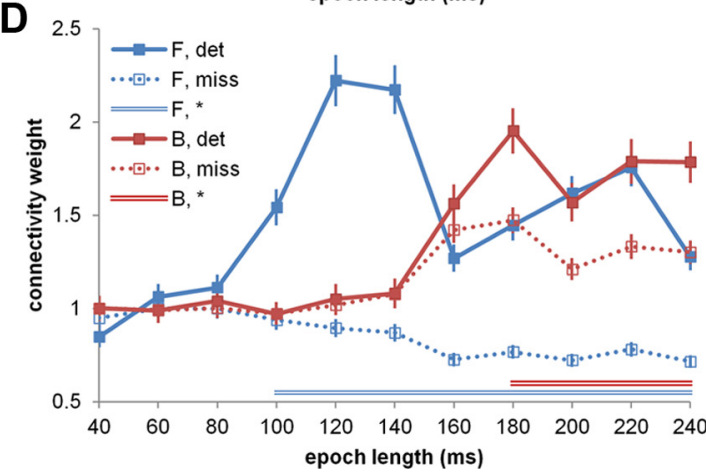

Figure 3. DCM. A, Structural DCMs. Individual dipole locations representing CSI and CSII/iSII were used to construct a structural model with three patterns of modulation by stimulus detection, representing feedforward, feedback, and recurrent processing between CSI and CSII. B, Model evidence. Models were compared using BMS for 11 data segments (plotted log-evidence difference against null model). C, Relative model evidence. Both recurrent and feedforward models explained data equally well for data segments $<120 \mathrm{~ms}$ (log-evidence difference $<5$ ). For longer data segments $(>140 \mathrm{~ms})$, the recurrent model was winning in all comparisons (very strong evidence $>5$; dashed gray line). $\boldsymbol{D}$, Connection strength across data segments. The feedforward connection (CSI- CSII, shown in blue) was stronger (100\% posterior probability) after aware stimulus detection for data segments $>80 \mathrm{~ms}$ (BMA, fixed effects). The feedback connection (cSII- CSI, shown in red) was stronger after detection for longer data epochs ( $>160 \mathrm{~ms}, 100 \%$ posterior probability), although the relative strength differences between conditions were less pronounced than for the feedforward connection. F, Forward; B, backward; det, detected; miss, missed. Asterisk marks significance. 

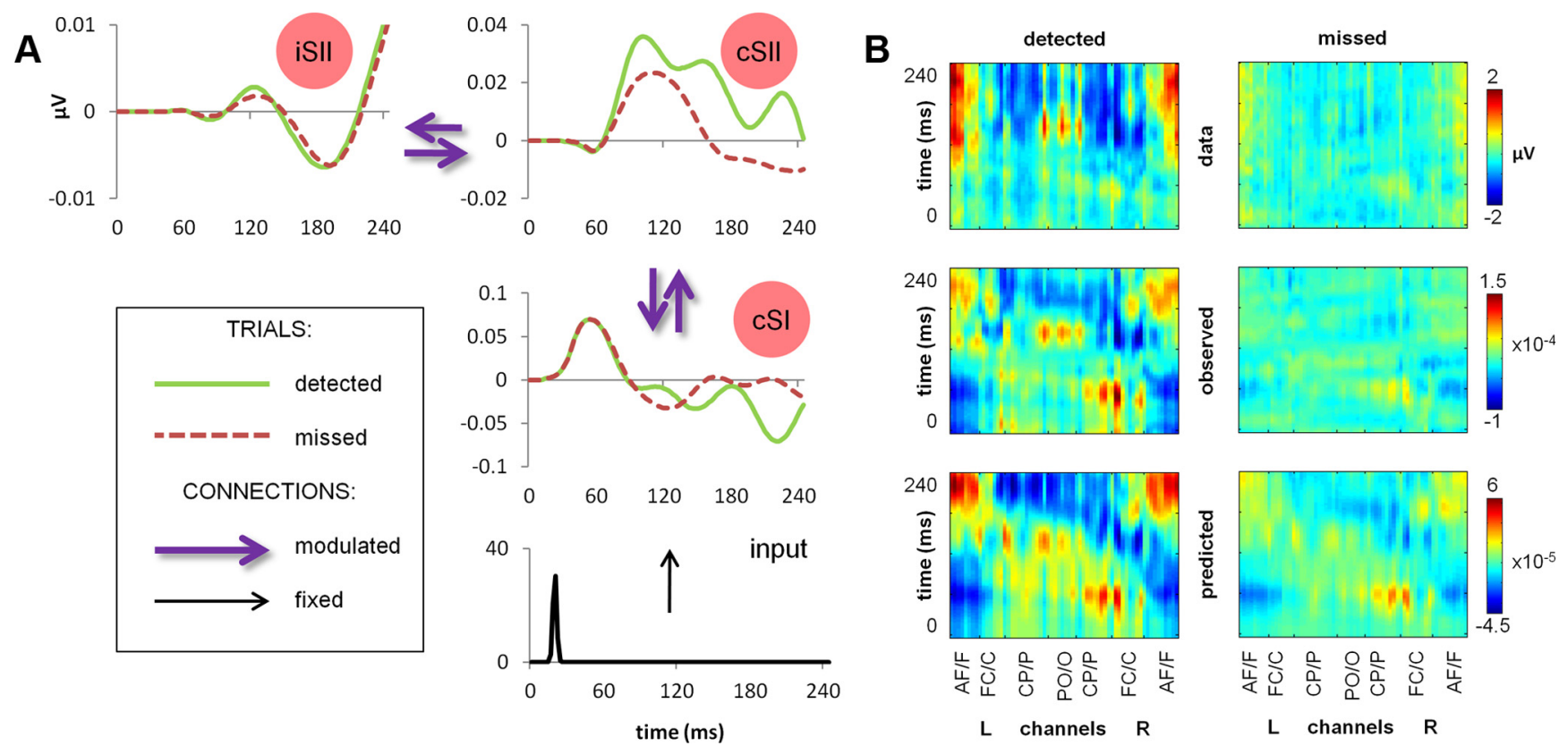

Figure 4. DCM output of the recurrent model. $\boldsymbol{A}$, Source activity estimates. Summary of the mean estimated activity at dipole level in the winning (recurrent) model $(n=19)$. $B$, Comparison of data and DCM output. Grand-averaged ERPs ( $n=19$ ) at all channels for detected (left column) and missed (right column) stimuli, plotted as (1) scalp data (top row), (2) DCM-observed responses, projected from scalp data onto eight spatial modes accounting for the greatest amount of explained variance and backprojected into channel space (middle row), and (3) DCM-predicted responses of the recurrent model, backprojected into channel space (bottom row). L, Left; R, right; $\mathrm{AF} / \mathrm{F}$, anteriofrontal/frontal; $\mathrm{FC} / \mathrm{C}$, frontocentral/central; $\mathrm{CP} / \mathrm{P}$, centroparietal/parietal; $\mathrm{PO} / \mathrm{O}$, parietooccipital/ occipital.

segments (140 ms and longer), the recurrent model was the winning model in all comparisons (very strong evidence $>5$; $\mathrm{cf}$. Penny et al., 2004; Fig. 3C).

Inspection of the specific dynamics of connection strength for detected and missed stimuli showed that the connection from cSI to cSII, for data segments longer than $80 \mathrm{~ms}$, was stronger for detected stimuli than when the stimulus had been missed $(n=$ 19, BMA, fixed effects; second-level $t$ tests, Bonferroni's corrected, $p<0.001$; Fig. $3 D$ ). The feedback connection from cSII to cSI was stronger for detected stimuli at longer latencies $(>160$ ms; $n=19$, BMA, fixed effects; second-level $t$ tests, Bonferroni's corrected $p<0.001$; Fig. $3 D$ ), although its relative strength increase was less pronounced than for the feedforward connection. Together, the analysis of time periods $>100 \mathrm{~ms}$ indicates that stimulus detection was characterized by a joint increase of feedforward and feedback connectivity and was best described by a model including recurrent processing within the somatosensory system.

To enable comparison of the modeling results to the actual data in compatible timescales, source activity estimates have been inspected ( $n=19$, averaged across participants; Fig. $4 A$ ), showing early cSI activity peaking independent of detection at $\sim 60 \mathrm{~ms}$ and a slowly diverging detection-related activity in CSII (peaking $\sim 100$ ms after stimulus), followed by a later divergence in cSI, most apparent toward the end of the analyzed epoch. Finally, an overall comparison of the initial scalp data to ERP responses as predicted by the recurrent model (backprojected into channel space; Fig. $4 B$ ) showed that the main neural responses for both detected and missed stimuli across all channels could be effectively explained by the model with the highest log evidence.

\section{Discussion}

To investigate the role of recurrent neural processing in somatosensory awareness, we applied DCM to EEG data recorded in a tactile perithreshold stimulus detection task. We found that ERPs associated with detected and missed stimuli diverged only after $\sim 80$ ms following stimulus presentation. Although early ERPs were well explained by a model assuming only modulation in the feedforward connectivity between cSI and cSII, recurrent processing in the model was needed to explain the effect of awareness on ERPs for data segments later than $140 \mathrm{~ms}$. Focusing on the recurrent model, the feedforward connection from cSI to cSII was stronger after stimulus detection for data segments longer than $80 \mathrm{~ms}$. Also the backward connection was modulated by detection, at least when later ERP components (>140 ms) were analyzed. Based on these findings, we conclude that increased recurrent processing within the somatosensory system, dominated by an enhanced cSI-cSII connection, underlies somatosensory detection.

\section{Early feedforward activation}

Several aspects of our results speak in favor of an early feedforward sweep after both detected and undetected stimulation (Lamme and Roelfsema, 2000). First of all, the similar dynamics of early ERPs characteristic for detected and missed stimuli are consistent with most previous studies on the timing of earliest observable neural responses associated with conscious processing of tactile or visual stimuli (Schubert et al., 2006; Del Cul et al., 2007; Wilenius and Revonsuo, 2007; Fahrenfort et al., 2008; but see Palva et al., 2005). Previous analyses of the spatiotemporal profile of late differential EEG responses in masking paradigms suggested that, although masking effectively disrupts recurrent processing, the feedforward sweep of activation is preserved (Del Cul et al., 2007; Fahrenfort et al., 2007; Koivisto and Revonsuo, 2010; van Gaal and Lamme, 2011; but see Khoe et al., 2004). Applying the same logic to our results, the similarities in early EEG possibly reflect the feedforward activation, whereas the late divergence of ERPs mark the feedback from higher sensory areas.

Second, our feedforward and recurrent models fitted equally well to early segments of the EEG data, suggesting that the feed- 
forward connections were sufficient to explain the early data epochs (but see Nicolelis et al., 1998, Liang et al., 2011 for alternative accounts based on parallel processing). It should be noted that, although such evidence does not allow for a conclusion that recurrent activity is absent in short epochs, it indicates that the effects of recurrent activity only contribute to explaining differences in longer EEG segments.

Finally, the cSI-cSII connection in our recurrent model showed detection-related activation only when data segments longer than $80 \mathrm{~ms}$ were analyzed, suggesting that, for shorter epochs, the feedforward sweep of activity from cSI to cSII is similar for both detected and missed stimuli. This can be further illustrated by inspecting source activity estimates of the recurrent model (Fig. $4 A$ ), in which cSI showed early sweep of activation, peaking independent of stimulus detection at $\sim 60 \mathrm{~ms}$. At approximately the same latency, cSII started to display a diverging activity pattern for detected and missed stimuli, which in turn drove later divergence in cSI. The timing of this activation was consistent with results of a previous MEG/modeling study (Jones et al., 2007), in which the first SI responses predicting detection were observed after $\sim 70 \mathrm{~ms}$, followed by additional detection-related peaks at $\sim 100$ and $\sim 135 \mathrm{~ms}$. Modeling the data with a biologically realistic computational SI model indicated that M70 reflects exogenous excitatory input reaching the supragranular layer of SI, possibly resulting from feedback signals from SII (Jones et al., 2007). Our results, together with those of Jones et al. (2007), suggest that $\sim 50 \mathrm{~ms}$ is enough to observe evidence for reentrant activity in the data (from the earliest observable stimulus-related response in cSI at $\sim 20 \mathrm{~ms}$ ), setting the upper boundary for duration of a full recurrent loop between cSI and cSII.

\section{Recurrent processing and stimulus detection}

Crucially, our DCM results on relative model evidence between recurrent and feedforward models support the hypothesis that EEG signals arising after aware detection of a tactile stimulus can be explained by increased recurrent activity within the somatosensory network in the later stages of stimulus processing. Such short-range recurrent interactions, through carrying a stable representation of sensory signals, are thought to underlie phenomenal awareness (Lamme, 2006) or preconscious states (Dehaene et al., 2006) and are considered a necessary condition for a more widespread, global recurrent activity associated with access consciousness and reportability (Dehaene et al., 2006; Lamme, 2006). Similar to our findings, recurrent processing between visual cortical areas V1 and V5 has been found to be crucial for awareness of visual motion (Pascual-Leone and Walsh, 2001; Silvanto et al., 2005; but see Koivisto et al., 2010).

Theoretical accounts of consciousness differ with respect to the relation between objective detection and subjective awareness of stimuli (Block, 2007). Although tasks using perithreshold stimulation are widely used as an operationalization of perceptual awareness (Dehaene and Changeux, 2011) and objective detection measures, among other forms of stimulus reportability, have been placed at the conjunction of attention and awareness (Dehaene et al., 2006; Lamme, 2006), several authors distinguished between objective and subjective measures of awareness (Libet et al., 1991; Crick and Koch, 2003; Lau and Passingham, 2006). Because in the current paradigm we assessed stimulus reportability in objective terms, our conclusions regarding the role of recurrent processing in conscious somatosensation are confined to stating its involvement in perceptual success. It remains to be tested whether recurrent activity can directly differentiate between, for example, subjective confidence levels of tactile perception. Nevertheless, a broadly distributed P300 componentreproducibly observed to strongly correlate with subjective measures of awareness (for a review, see Dehaene and Changeux, 2011) — was clearly present in our data (Fig. 2C,D), suggesting that our behavioral measure was sensitive to the participants' perceptual awareness of the stimulus.

It should be further noted that explaining perceptual processing primarily in terms of feedforward and feedback connections does not rule out a possible influence of horizontal connections within cortical regions. Horizontal connections are thought to play a crucial role in perceptual grouping (for review, see Roelfsema, 2006), and visual contextual effects on late ERP divergence tend to be explained in terms of horizontal neuronal influences (Khoe et al., 2004). In the somatosensory cortex, horizontal connections have been mostly studied in rat barrel cortex and hypothesized to subserve within-column excitation and surround input integration, although their precise functional role remains unknown (Boucsein et al., 2011). On a higher level, lateral architectures of interconnected areas have been speculated to be sufficient for phenomenal awareness (Malach, 2007); however, the involvement of higher areas in aware perception is still a matter of debate (Del Cul et al., 2009).

So far, evidence for the notion that perceptual consciousness arises through recurrent loops of activity between brain areas (Lamme, 2000, 2006) has been primarily limited to studies of vision (Pascual-Leone and Walsh, 2001; Ro et al., 2003; Silvanto et al., 2005; Boehler et al., 2008; Fahrenfort et al., 2008), with an exception of a recent DCM/EEG study in which auditory stimuli were delivered to patients with severe disorders of consciousness (Boly et al., 2011). Our findings allow for a generalization of the recurrent processing hypothesis to the somatosensory modality, suggesting that recurrent activity between regions involved in stimulus processing might be a general mechanism underlying perceptual awareness.

\section{References}

Allison T, McCarthy G, Wood CC, Jones SJ (1991) Potentials evoked in human and monkey cerebral cortex by stimulation of the median nerve. Brain 114:2465-2503.

Benjamini Y, Hochberg Y (1995) Controlling the false discovery rate: a practical and powerful approach to multiple testing. J R Stat Soc Series B Stat Methodol 57:289-300.

Block N (2007) Consciousness, accessibility, and the mesh between psychology and neuroscience. Behav Brain Sci 30:481-548.

Boehler CN, Schoenfeld MA, Heinze HJ, Hopf JM (2008) Rapid recurrent processing gates awareness in primary visual cortex. Proc Natl Acad Sci U S A 105:8742-8747.

Boly M, Garrido MI, Gosseries O, Bruno MA, Boveroux P, Schnakers C, Massimini M, Litvak V, Laureys S, Friston K (2011) Preserved feedforward but impaired top-down processes in the vegetative state. Science 332:858-862.

Boucsein C, Nawrot MP, Schnepel P, Aertsen A (2011) Beyond the cortical column: abundance and physiology of horizontal connections imply a strong role for inputs from the surround. Front Neurosci 5:32.

Crick F, Koch C (2003) A framework for consciousness. Nat Neurosci 6:119-126.

David O, Kiebel SJ, Harrison LM, Mattout J, Kilner JM, Friston KJ (2006) Dynamic causal modeling of evoked responses in EEG and MEG. Neuroimage 30:1255-1272.

Dehaene S, Changeux JP (2011) Experimental and theoretical approaches to conscious processing. Neuron 70:200-227.

Dehaene S, Changeux JP, Naccache L, Sackur J, Sergent C (2006) Conscious, preconscious, and subliminal processing: a testable taxonomy. Trends Cogn Sci 10:204-211.

Del Cul A, Baillet S, Dehaene S (2007) Brain dynamics underlying the nonlinear threshold for access to consciousness. PLoS Biol 5:e260. 
Del Cul A, Dehaene S, Reyes P, Bravo E, Slachevsky A (2009) Causal role of prefrontal cortex in the threshold for access to consciousness. Brain 132:2531-2540.

de Lafuente V, Romo R (2006) Neural correlate of subjective sensory experience gradually builds up across cortical areas. Proc Natl Acad Sci U S A 103:14266-14271.

Disbrow E, Roberts T, Poeppel D, Krubitzer L (2001) Evidence for interhemispheric processing of inputs from the hands in human S2 and PV. J Neurophysiol 85:2236-2244.

Eickhoff SB, Stephan KE, Mohlberg H, Grefkes C, Fink GR, Amunts K, Zilles K (2005) A new SPM toolbox for combining probabilistic cytoarchitectonic maps and functional imaging data. Neuroimage 25:1325-1335.

Fahrenfort JJ, Scholte HS, Lamme VAF (2007) Masking disrupts reentrant processing in human visual cortex. J Cogn Neurosci 19:1488-1497.

Fahrenfort JJ, Scholte HS, Lamme VAF (2008) The spatiotemporal profile of cortical processing leading up to visual perception. J Vis 8:1-12.

Garrido MI, Kilner JM, Kiebel SJ, Friston KJ (2007) Evoked brain responses are generated by feedback loops. Proc Natl Acad Sci U S A 104: 20961-20966.

Hagiwara K, Okamoto T, Shigeto H, Ogata K, Somehara Y, Matsushita T, Kira J, Tobimatsu S (2010) Oscillatory gamma synchronization binds the primary and secondary somatosensory areas in humans. Neuroimage 51:412-420.

Jones SR, Pritchett DL, Stufflebeam SM, Hämäläinen M, Moore CI (2007) Neural correlates of tactile detection: a combined magnetoencephalography and biophysically based computational modeling study. J Neurosci 27:10751-10764.

Khoe W, Freeman E, Woldorff MG, Mangun GR (2004) Electrophysiological correlates of lateral interactions in human visual cortex. Vision Res 44:1659-1673.

Kiebel SJ, Daunizeau J, Phillips C, Friston KJ (2008) Variational Bayes inversion of the equivalent current dipole model in EEG/MEG. Neuroimage 39:728-741.

Koivisto M, Revonsuo A (2010) Event-related brain potential correlates of visual awareness. Neurosci Biobehav Rev 34:922-934.

Koivisto M, Mäntylä T, Silvanto J (2010) The role of early visual cortex (V1/V2) in conscious and unconscious visual perception. Neuroimage $51: 828-834$.

Lamme VAF (2000) Neural mechanisms of visual awareness: a linking proposition. Brain Mind 1:385-406.

Lamme VAF (2006) Towards a true neural stance on consciousness. Trends Cogn Sci 10:494-501.

Lamme VAF, Roelfsema PR (2000) The distinct modes of vision offered by feedforward and recurrent processing. Trends Neurosci 23:571-579.

Lau HC, Passingham RE (2006) Relative blindsight in normal observers and the neural correlate of visual consciousness. Proc Natl Acad Sci U S A 103:18763-18768

Liang M, Mouraux A, Iannetti GD (2011) Parallel processing of nociceptive and non-nociceptive somatosensory information in the human primary and secondary somatosensory cortices: evidence from dynamic causal modeling of functional magnetic resonance imaging data. J Neurosci 31:8976-8985.
Libet B, Alberts WW, Wright EW Jr, Feinstein B (1967) Responses of human somatosensory cortex to stimuli below threshold for conscious sensation. Science 158:1597-1600.

Libet B, Pearl DK, Morledge DE, Gleason CA, Hosobuchi Y, Barbaro NM (1991) Control of the transition from sensory detection to sensory awareness in man by the duration of a thalamic stimulus. Brain 114:1731-1757.

Malach R (2007) The measurement problem in consciousness research. Behav Brain Sci 30:516-517.

Meador KJ, Ray PG, Echauz JR, Loring DW, Vachtsevanos GJ (2002) Gamma coherence and conscious perception. Neurology 59:847-854.

Nicolelis MAL, Ghazanfar AA, Stambaugh CR, Oliveira LMO, Laubach M, Chapin JK, Nelson RJ, Kaas JH (1998) Simultaneous encoding of tactile information by three primate cortical areas. Nat Neurosci 1:621-630.

Palva S, Linkenkaer-Hansen K, Näätänen R, Palva JM (2005) Early neural correlates of conscious somatosensory perception. J Neurosci 25:52485258.

Pascual-Leone A, Walsh V (2001) Fast backprojections from the motion to the primary visual area necessary for visual awareness. Science 292:510-512.

Penny WD, Stephan KE, Mechelli A, Friston KJ (2004) Comparing dynamic causal models. Neuroimage 22:1157-1172.

Ray PG, Meador KJ, Smith JR, Wheless JW, Sittenfeld M, Clifton GL (1999) Physiology of perception: cortical stimulation and recording in humans. Neurology 52:1044-1049.

Ro T, Breitmeyer B, Burton P, Singhal NS, Lane D (2003) Feedback contributions to visual awareness in human occipital cortex. Curr Biol 11:1038-1041.

Roelfsema PR (2006) Cortical algorithms for perceptual grouping. Annu Rev Neurosci 29:203-227.

Schubert R, Blankenburg F, Lemm S, Villringer A, Curio G (2006) Now you feel it-now you don't: ERP correlates of somatosensory awareness. Psychophysiology 43:31-40.

Silvanto J, Lavie N, Walsh V (2005) Double dissociation of V1 and V5/MT activity in visual awareness. Cereb Cortex 15:1736-1741.

Stephan KE, Penny WD, Daunizeau J, Moran RJ, Friston KJ (2009) Bayesian model selection for group studies. Neuroimage 46:1004-1017.

Stephan KE, Penny WD, Moran RJ, den Ouden HEM, Daunizeau J, Friston KJ (2010) Ten simple rules for dynamic causal modeling. Neuroimage 49:3099-3109.

Thees S, Blankenburg F, Taskin B, Curio G, Villringer A (2003) Dipole source localization and fMRI of simultaneously recorded data applied to somatosensory categorization. Neuroimage 18:707-719.

Tononi G, Koch C (2008) The neural correlates of consciousness: an update. Ann N Y Acad Sci 1124:239-261.

van Gaal S, Lamme VAF (2011) Unconscious high-level information processing: implication for neurobiological theories of consciousness. Neuroscientist. Advance online publication. Retrieved December 8, 2011. doi:10.1177/1073858411404079.

Wilenius ME, Revonsuo AT (2007) Timing of the earliest ERP correlate of visual awareness. Psychophysiology 44:703-710. 